\title{
Grand Challenges in Text Entry
}

Per Ola Kristensson

University of St Andrews

St Andrews, UK

pok@st-andrews.ac.uk

Stephen Brewster

University of Glasgow

Glasgow, UK

stephen.brewster@glasgow.ac.uk

\section{James Clawson}

Georgia Institute of Technology

Atlanta, Georgia, USA

jamer@cc.gatech.edu

\section{Mark Dunlop}

University of Strathclyde

Glasgow, UK

mark.dunlop@cis.strath.ac.uk

\section{Leah Findlater}

University of Maryland

College Park, Maryland, USA

leahkf@umd.edu

\section{Poika Isokoski}

University of Tampere

Tampere, Finland

poika.isokoski@uta.fi

\section{Benoît Martin}

University of Lorraine

Metz, France

benoit.martin@univ-lorraine.fr

\section{Antti Oulasvirta}

Max Planck Institute for Informatics

Saarbrücken, Germany

antti.oulasvirta@mpi-inf.mpg.de

\section{Keith Vertanen}

Montana Tech

Butte, Montana, USA

kvertanen@mtech.edu

\section{Annalu Waller}

University of Dundee

Dundee, UK

awaller@computing.dundee.ac.uk

Copyright is held by the author/owner(s).

CHI 2013 Extended Abstracts, April 27-May 2, 2013, Paris, France.

ACM 978-1-4503-1952-2/13/04.

\begin{abstract}
Our workshop serves two purposes. First, to bring text entry researchers working in the human-computer interaction (HCI), natural language processing (NLP) and augmentative and alternative communication (AAC) communities together at CHI. Second, we will set three major grand challenges for text entry research: a) removing the performance bottleneck in text entry; b) designing efficient localized text entry methods; and c) bridging the communication gap between users with disabilities and society at large. These challenges will be discussed in a panel format at the workshop. The discussions will center on support activities, such as identifying obstacles for success in meeting these challenges and formalizing procedures for measuring progress in the text entry field.
\end{abstract}

\section{Keywords}

Text entry; augmentative and alternative communication; accessibility; internationalization

\section{ACM Classification Keywords}

H.5.2 [Information interfaces and presentation]: User Interfaces-Input devices and strategies

\section{Introduction}

Text entry methods are critical in our society, both for preserving our culture and for enabling us to

communicate with each other at a distance. For 
nonspeaking individuals, text entry methods can provide a critical communication capability.

As an active research field, the last decades have seen tremendous progress in devising faster and more fluid text entry methods. Methods have been created for a variety of different user groups, devices and situations, such as mobile systems (e.g. [2]), games (e.g. [10]), and users with motor impairments (e.g. [11]). See [1, $3,6,7,12]$ for recent text entry surveys.

However, while tremendous progress has been made in text entry, the research community is scattered across different fields, such as human-computer interaction (HCI), natural language processing (NLP), speech processing, and augmentative and alternative communication (AAC). This has resulted in many researchers being unaware of progress made in neighboring fields. To address this issue, this workshop aims to bring together text entry researchers from diverse research fields and center text entry research activities at $\mathrm{CHI}$

Another important aim with this workshop is to strengthen text entry research by focusing on three clearly defined grand challenges for text entry: a) removing the performance bottleneck in text entry; b) designing efficient localized text entry methods; and c) bridging the communication gap between users with disabilities and society at large.

The workshop will be organized as a series of three panel discussions with each panel tackling one of the above grand challenges. The discussions will center on support activities, such as identifying obstacles for success in meeting these challenges and formalizing procedures for measuring progress in text entry.

\section{Experiences from the CHI 2012 workshop}

Six of the ten co-organizers for this workshop proposal were also involved in organizing the $\mathrm{CHI} 2012$ workshop Designing and evaluating text entry methods $[5]^{1}$. The purpose of that workshop was: a) to build a community, b) to promote $\mathrm{CHI}$ as a natural and compelling focal point for all kinds of text entry research, and c) to discuss some difficult issues that are hard or near impossible to handle within the traditional format of research papers.

At our workshop at CHI 2012 we received 17 submissions and accepted 13 for an acceptance rate of $76 \%$. Each submission was assigned 2-4 reviewers and all submissions were discussed at a program committee meeting. Only papers we judged making substantia contributions to the topics of the workshop were accepted. All accepted papers were discussed in four different informal panels. The previous workshop was successful in bringing new people to $\mathrm{CHI}$ from the AAC community (three accepted papers) and in bringing new HCI people to the text entry field (one accepted paper from the design community and two accepted papers from the systems and tools community).

\section{Grand challenges in text entry}

For CHI 2013, we want to leverage the momentum of the successful community-building efforts initiated at the CHI 2012 workshop. In the previous workshop it became apparent that clearly identified research challenges could help unify, strengthen and focus text entry research.

Therefore, for CHI 2013 we have identified three grand challenges that will form the themes of three in-depth

\footnotetext{
${ }^{1}$ http://textentry.org/chi2012/
} 
panel discussions. The grand challenges for text entry that will be discussed are:

\section{- Removing the performance bottleneck of text} entry: Text entry systems are limited in performance, and this in turn limits users' communication speeds. This problem is most acute for mobile text entry methods $[1,2,7,12]$, but the limited performance potential of text entry also holds true for regular fullsized keyboards. For example, while humans can speak at 200 wpm [8], average text entry rates on users' own familiar full-sized keyboards are circa 70 wpm [4]. The challenge here is to see if there are new input devices, interfaces, or recognition techniques that can significantly outperform state-of-the-art. We believe competition on some common device form factors and input/output modalities would help further the field.

\section{- Designing efficient localized text entry}

methods: Despite the world's tremendously diverse

writing systems and languages, the QWERTY keyboard

layout-designed according to character bigram

frequencies in the English language-is still pervasive

all over the world. However, the QWERTY layout is

clearly a suboptimal solution for many European

languages as well as for languages with large character

sets such as Chinese, Japanese, and Sinhalese and

other Indic scripts. Yet, advanced research on new

efficient localized text entry methods is rare. On the

surface this may appear surprising. However, it can be explained by the lack of support and resources for working with less mainstream languages. Many efficient text entry methods require high-quality language models and these are difficult to build without access to representative text corpora and expertise in statistical language modeling. Further, the design challenge posed by enabling users to input characters from potentially very large character sets is difficult to tackle and currently no optimal solutions exist.

- Bridging the communication gap between users with disabilities and society at large: Users with disabilities, such as nonspeaking individuals, often rely on text entry methods for their primary

communication needs. Users with certain needs have to rely on suboptimal text entry methods, such as singleswitch scanning systems or predictive keyboards. The grand challenge here is to reduce the communication entry rate gap between users of such systems and society at large. This is an extremely difficult problem but we believe a friendly yet competitive approach that involves standardized test procedures may enable the community to identify text entry methods that can substantially surpass state-of-the-art.

\section{Support activities}

The workshop will be structured as a series of three panel discussions. Each panel will tackle one of the above grand challenges, identifying obstacles for success and formalizing procedures for measuring progress.

For example, for the challenge of removing the performance bottleneck of text entry, it will be vital to discuss experimental protocols that ensure text entry methods are evaluated and compared in a systematic and replicable way. Currently there is no universally accepted experimental procedure for evaluating text entry performance. However, we believe it will be critical to develop such procedures if we are to ensure we are all moving towards the same goal.

For the challenge designing efficient localized text entry methods, it will be critical to provide support to 
researchers in the form of open high-quality text corpora for different languages and tools that simply the creation of language models suitable for text entry.

For the challenge on bridging the communication gap between users with disabilities and society at large, shared resources, such as language models, can help progress. For this challenge, standardized test procedures are less likely to be helpful, due to the diversity of users' needs and capabilities. Here, new interdisciplinary collaborations between user-centered designers and researchers with expertise on machine learning and language modeling could lead to radically new and efficient solutions.

\section{Conclusions}

Last year we initiated a move to unify text entry researchers scattered across different research fields and to create an interdisciplinary text entry community centered at $\mathrm{CHI}$. We attracted high-quality submissions and we brought in researchers active outside of the $\mathrm{HCI}$ field to $\mathrm{CHI}$. We want to leverage this momentum and ensure we can create a sustainable interdisciplinary text entry community at $\mathrm{CHI}$

However, we also want to ensure text entry research realizes its full impact in our society. Therefore, this workshop sets three major grand challenges for text entry research. The panel discussions will center on support activities, such as identifying obstacles for success in meeting these challenges and formalizing procedures for measuring progress in the text entry field. We believe this will help unify, strengthen and focus text entry research.

\section{References}

[1] Dunlop, M.D. and Masters, M.M. Pickup usability dominates: a brief history of mobile text entry research and adoption. International Journal of Mobile Human Computer Interaction 1, 1 (2009), 42-59.

[2] Goel, M., Findlater, L., and Wobbrock, J. WalkType: using accelerometer data to accomodate situational impairments in mobile touch screen text entry. In Proc. CHI 2012, ACM Press (2012), 2687-2696.

[3] Kristensson, P.O. Five challenges for intelligent text entry methods. AI Magazine 30, 4 (2009), 85-94.

[4] Kristensson, P.O. and Vertanen, K. 2012. Performance comparisons of phrase sets and presentation styles for text entry evaluations. In Proc. IUI 2012. ACM Press (2012), 29-32.

[5] Kristensson, P.O, Clawson, J., Dunlop, M., Isokoski P., Roark, B., Vertanen, K., Waller, A. and Wobbrock J.O. 2012. Designing and evaluating text entry methods. In Ext. Abstr. CHI 2012, ACM Press (2012), 2747-2750.

[6] MacKenzie, I. S., and Tanaka-Ishii, K. 2007. Text Entry Systems. Morgan Kauffman.

[7] MacKenzie, I. S., and Soukoreff, R.W. Text entry for mobile computing: models and methods, theory and practice. Human-Computer Interaction 17, 2 (2002), 147-198.

[8] Rosenbaum, D.A. 1991. Human Motor Control. Academic Press.

[9] Ward, D. J., and MacKay, D. J. C. Fast hands-free writing by gaze direction. Nature 418 (2002), 838.

[10] Wilson, A.D. and Agrawala, M. Text entry using a dual joystick game controller. In Proc. CHI 2006, ACM Press (2006), 475-478.

[11] Wobbrock, J.O. and Myers, B.A. Trackball text entry for people with motor impairments. In Proc. $\mathrm{CHI}$ 2006, ACM Press (2006), 479-488.

[12] Zhai, S., Kristensson, P.O. and Smith, B.A. In search of effective text input interfaces for off the desktop computing. Interacting with Computers 17, 3 (2005), 229-250. 